\title{
Ashwaganda, Rhodiola rosea and Maca as Adaptogens
}

ISSN: 2637-7659

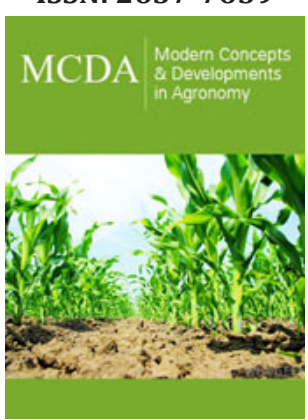

*Corresponding author: Suzan Tireki, Assistant Professor, Food Engineering Department, Istanbul Sabahattin Zaim University, 34303, Istanbul, Turkey

Submission: 㘹 May 31, 2021

Published: 侮June 14, 2021

Volume 8 - Issue 5

How to cite this article: Suzan Tireki. Ashwaganda, Rhodiola rosea and Maca as Adaptogens. Mod Concep Dev Agrono. 8(5). MCDA. 000696. 2021. DOI: 10.31031/MCDA.2021.08.000696

Copyright@ Suzan Tireki. This article is distributed under the terms of the Creative Commons Attribution 4.0 International License, which permits unrestricted use and redistribution provided that the original author and source are credited.

\section{Suzan Tireki*}

Assistant Professor, Food Engineering Department, Istanbul Sabahattin Zaim University, Turkey

\begin{abstract}
Adaptogens are non-toxic plant-based substances which help balance, restore, protect the body from the harmful stress effects. They are used to calm, energize, counteract stress and improve mood, mental clarity, keep fit or decrease disorders based on aging such as lack of attention. They can also show antioxidant, immunomodulator, hypoglycemic, nootropic, neuroprotective, antifatigue anxiolytic properties. Interest in adaptogens on the rise in foods and beverages because they are safe, economical and efficacious. It is anticipated that this increase will continue as consumers will seek food products improving mood and boosting brain health in the future. Adaptogens have the potential to serve for these purposes. Hence, this mini review addresses three of the most common adaptogens, namely Ashwaganda (Withania somnifera), Rhodiola rosea and Maca (Lepidium meyenii), which can be used in functional products improving the quality of life as proactive wellness and healthcare actions.
\end{abstract}

Keywords: Adaptogens; Ashwaganda; Withania somnifera; Rhodiola rosea; Maca; Lepidium meyenii

\section{Introduction}

Adaptogens are primarily plant-based agents which help balance, restore and improve the ability of body to resist and be protected from the harmful impacts of stress and stress-related disorders [1,2]. Adaptogens are harmless to the host; have a non-specific effect and ability to have a role as general normalizer/stabilizer [2]. These special class of plants assists to calm and energize in a simultaneous way and have abilities helping counteract stress and improve mood, physical stamina and mental clarity [1]. Besides eliminating stress, adaptogens increase physical resistance, provide fitness and decrease disorders in connection with aging such as weakness, memory loss and lack of attention. They can enhance cognitive performance and have a role as immunomodulator, hypocholesterolemic, hypoglycemic and antioxidant [3]. It was reported that adaptogens depict antifatigue, antidepressive, neuroprotective and central nervous system stimulating properties in animals and isolated neuronal cells [4].

Considerable interest in adaptogens have been seen due to their cost effectiveness, efficacy and safety. Many scientific investigations have explained their safety and efficacy issues in human diseases based on experimental and clinical studies [2]. They are also used in functional foods and beverages, which have been led by proactive healthcare actions. Consumers are looking for brain function benefits such as focus, cognition and memory from the food products [5]. It is predicted that the attention to adaptogens will increase as consumers will be interested in the products boosting brain health and enhancing mood in the future [6], which can be provided by adaptogens. Therefore, adaptogens will be one of the remarkable future plant-based ingredients. Ashwaganda (Withania somnifera), Rhodiola rosea and Maca (Lepidium meyenii) are three of the most common adaptogens that are used in functional products (Table 1) helping people to enhance their life quality. 
Table 1: Some of the commercial products containing adaptogenic plants [22-24].

\begin{tabular}{|c|c|c|}
\hline Product & Adaptogenic plant & Brand \\
\hline Sparkling chai tea with ashwagandha and apple & Ashwaganda (Withania somnifera) & 8 \\
\hline Natural nootropic beverage (Focus-energy mood) & Rhodiola rosea & Nubrain Prodigy ${ }^{\text {th }}$ [23] \\
\hline $\begin{array}{c}\text { Non-sparkling organic probiotic water with maca, } \\
\text { pineapple and coconut }\end{array}$ & Maca (Lepidium meyenii) & Kamsa [24] \\
\hline
\end{tabular}

\section{Ashwaganda (Withania somnifera)}

Ashwagandha (Withania somnifera) belongs to the Solanaceae family and also known as "Indian winter cherry" or "Indian ginseng". It is one of the most popular and important plants in the tradition of the Indian Ayurvedic medicine for its many beneficial health activities [7]. It is classified as an adaptogen and in the subcategory of Medhyarasayanas, in which Medhya means intellectual capacity and memory [8]. Ashwagandha is used in the management of stress, elevation of energy and improvement of cognitive health [7]. It has the ability to decrease inflammation, cortisol, anxiety, blood sugar levels and depression [7,9]. Ashwagandha has been reported to have anti-stress, anti-cancer, anti-inflammatory, antihyperglycemic, antioxidant, immunostimulant, anti-epileptic, antidepressant, anti-diabetic, arthritic, anti-coagulant properties $[7,10]$ and palliative effects like regenerating, rejuvenating and growth enhancement [11].

Long tuberous roots, ovate and petiolate leaves, short stems and greenish bisexual and axillary flowers characterize erect, grey evergreen Ashwagandha. With twenty-nine common metabolites obtained from the leaves and root extracts, the flowers, stems, roots and leaves of the plant have therapeutic values [7,9]. Ashwagandha is generally sold as finely sieved powder that can be combined with water, ghee or honey. Alkaloids (anahygrine, cuseohygrine, anaferine, isopelletierine and others), saponins and steroidal lactones (withaferins, withanolides) are among the biologically active chemical components of Withania somnifera. Ashwagandha contains anti-stress agents called acylsterylglucosides and sitoindosides. Active components of this adaptogen, such as the sitoindosides VII-X and Withaferin-A, demonstrated to exhibit considerable anti-stress activity in acute stress models. Many of the components of Ashwagandha have immunomodulatory properties. Withasomniferin-A and 5-dehydroxy withanolide-R are obtained from the aerial portions of Withania somnifera [12].

\section{Rhodiola rosea}

Rhodiola rosea is a popular botanical adaptogen and also known as "rose root" or "golden root". It is in the Crassulaceae family. It is commonly used as a non-toxic natural health product with several beneficial properties like anti-diabetic, anti-cancer, anti-aging and cardio-protective due to the strong adaptogens found in the root extracts. Rhodiola rosea is the species that has been shown to be the safest for human use with no documented adverse effects. It has neuroprotective effects and generates an unspecific resistance to fatigue, depression, stress and exercise performance $[13,14]$. Like ashwagandha, Rhodiola rosea increases the resilience of body to stressors by impacting the axis of hypothalamic-pituitary-adrenal and protects against mental and physical exhaustion due to stress. Military people and athletes used this adaptogenic herb to boost their stress resistance and combat fatigue [1]. Rhodiola rosea was found to improve physiological features including cardioprotective effects, central nervous system simulation and cognitive abilities like memory, learning and attention. In the United States and Europe, it has been very popular due to its antioxidant, anxiolytic and antidepressant benefits [14].

Rhodiola rosea can be found throughout Central and Northern Europe's mountainous regions and North America and Asia [15]. Rhodiola species are perennials with robust, woody multicipidal caudices that are typically branched and covered with thick appressed leaves. Male flowers of the plant are yellow while the female flowers are yellow to red. Young plants have mostly hairy roots with a little rhizome during their first two years of growth and root generation starts after 3-4 years. The above-ground portions are flowering/non-flowering stems while the underground components are $10-25 \mathrm{~cm}$ long roots and a $20-50 \mathrm{~cm}$ diameter rhizome. The roots attain commercial size of 0.5-1.0 kilogram after 4 growing years of culture [16].

Rhodiola rosea's therapeutic activities are related to both the existence of a complex mixture of active components and their relative composition. There are also many variations in the content of the plant depending on the cultivation geography and extraction technique [14]. Flavonoids, tyrosol, proanthocyanidines and cinnamyl alcohol are the polyphenols found in Rhodiola plants. They also contain glycosides, essential oils, sugars, proteins, fats, alcohols and organic acids. Rhodiola rosea has a polyphenol content around $41.4 \pm 3.41 \%$. Primary components of Rhodiola rosea are salidroside and its aglycone, tyrosol and the amount of the two compounds is frequently employed as a parameter for the evaluation of crude Rhodiola rosea drugs [17]. Rhodiola rosea extracts have been applied in beverages (Table 1), food additives and commercial medicinal preparations globally [15].

\section{Maca (Lepidium meyenii)}

Maca (Lepidium meyenii) is a cruciferous plant native to the Andes of Peru. It belongs to Brassicaceae family. It has been utilized as an adaptogen to treat infertility, anemia, rheumatism, respiratory disorders and female hormone balance for centuries [18,19]. There has been an increase in attention to maca-based products since the 1990s [20] due to its health benefits. Health claims of improving 
vitality and longevity, promoting fertility and libido and reducing menopausal symptoms in women have led to maca being marketed as a superfood [19]. Anti-oxidation, anti-fatigue, neuroprotection, immunomodulation, anti-cancer, anti-bacterial activity and improved digestion system and skin health functions are also maca's bioactivities [21].

Hypocotyls and main tap root are the edible portions of maca. Maca's flower, leaf and stem as source edible vegetables are underused. Maca root can grow to be around $20 \mathrm{~cm}$ in circumference. Plant can grow to be about $10-20 \mathrm{~cm}$ height. Shape of maca roots depends on genetic variability, which is specified by different weights from $1 \mathrm{~kg}$ to $5 \mathrm{~kg}$, shapes (spherical, oval, spindleshaped, spherical oval) and colour (white, purple, claret, cream, red, orange, yellow). Nutritional composition also depends on the genetic variability. Principal dietary components of maca root are starch, dietary fiber and protein with $4.63-10.40 \%$ moisture content. Dietary fibers, vitamins, minerals and vital amino acids are also found in the leaves. Maca's minor nutritional ingredients are thought to play a role in its biological advantages [21]. Polyphenols, malamedas, non-starch polysaccharides, macaenes, macahydantoins, glucosinolates and macamides are among the nutritional and pharmacological components found in maca roots and leaves [19,21]. Maca can be used in market products (Table 1) [22-24] like pills, capsules, flour, liquor, chocolate and beverages. In addition, ingredients derived from maca have high potential as food additives for certain properties such as texturizing and antioxidant [21].

\section{Conclusion}

Food products formulated with adaptogenic plants have gained interest for consumers who want to improve their life quality due to their health benefits. This attention will continue in these products especially for some of the adaptogens like Ashwaganda (Withania somnifera), Rhodiola rosea and Maca (Lepidium meyenii) because of their cost, safety and efficaciousness in line with the consumer trends. Because future consumers will seek functional products improving mood boosting brain health and this can be served by adaptogens. Innovation and research efforts are needed for sustainable and standardized products in the current regulatory environment so that consumers can benefit from these niche products.

\section{Conflict of Interest}

The author declares that there is no conflict of interest.

\section{References}

1. Ankita W, Pranay W, Awani KR, Ruchi T, Sunil KP (2019) Adaptogens with a special emphasis on Withania somnifera and Rhodiola rosea. In: Nutrition and enhanced sports performance, ( $\left.2^{\text {nd }} e d n\right)$, Debasis B, Sreejayan N, Chandan KS (Eds.), Chapter 34, Oxford: Academic Press, Oxford, United Kingdom, pp: 407-418.

2. Arunabha R, Kavati G, Sana R, Nishant R, Rashmi A (2021) Role of nutraceuticals as adaptogens In: Nutraceuticals: Efficacy, safety and toxicity, (2 ${ }^{\text {nd }}$ edn), Ramesh CG, Rajiv L, Ajay S (Eds.), Chapter 16, Oxford: Academic Press, Oxford, United Kingdom, pp: 229-244.

3. Fulvio RM, Elisaldo AC (2007) Brazilian plants as possible adaptogens: An ethnopharmacological survey of books edited in Brazil. J Ethnopharmacol 109(3): 493-500.

4. Alexander P, Georg W (2010) Effects of adaptogens on the central nervous system and the molecular mechanisms associated with their stress-protective activity. Pharmaceuticals 3(1): 188-224.

5. Foodinsight.org (2020) 2020 Food and health survey.

6. Mintel.com (2020) Global food and drink trends.

7. Rinku D, Roukiah K, Ryan G, Shyam SM, Subhra M (2019) Withania somnifera (Ashwagandha) and Withaferin A: Potential oncology. Int J Mol Sci 20(21): 5310.

8. Dnyanrai C, Sauvik B, Sekhar B (2017) Efficacy and safety of Ashwagandha (Withania somnifera (L.) Dunal) root extract in improving memory and cognitive functions. J Diet Suppl 14(6): 599-612.

9. Mirjalili MH, Moyano E, Bonfill M, Cusido RM, Palazon J (2009) Steroidal lactones from Withania somnifera, an ancient plant for novel medicine. Molecules 14(7): 2373-2393.

10. Irene D (2019) An overview of functional beverages. In: Functional and medicinal beverages, The science of beverages, Alexandru MG, Alina MH (Eds.), Chapter 1, Duxford: Academic Press, United Kingdom, 11: 1-40.

11. Subbaraju GV, Vanisree M, Rao CV, Sivaramakrishna C, Sridhar P, et al. (2006) Ashwagandhanolide, a bioactive dimeric thiowithanolide isolated from roots of Withania somnifera. J Nat Prod 69(12): 17901792.

12. Narendra S, Mohit B, Prashanti DJ, Marilena G (2011) An overview on Ashwagandha: A rasayana (rejuvenator) of Ayurveda. Afr J Tradit Complement Alren Med 8(5 Suppl): 208-213.

13. Wei ling P, Meng ying Z, Ru yu B, Li kang S, Wen hua L, et al. (2020) Anti-inflammatory effects of Rhodiola rosea L.: A review. Biomed Pharmacother 121: 109552.

14. Paola S, Federica T, Paola L, Ranieri U, Urska V, et al. (2018) Rhodiola rosea, a protective antioxidant for intense physical exercise: An in vitro study. J Funct Foods 48: 27-36.

15. Ljuba E, Milka T, Daniela A, Jordanka S (2010) Chemical composition of the essential oils of Rhodiola rosea L. of three different origins. Pharmacogn Mag 6(24): 256-258.

16. Bertalan G, Zsuzsanna G, Eva H, Eva S, Vladimir V, et al. (2010) Importance and quality of roseroot (Rhodiola rosea L.) growing in the European North. Z Arznei-Gewurzpfla 15(4): 160-169.

17. Hsiu Mei C, Hsin Chun C, Chin Sheng W, Po Yuan W, Kuo Ching W (2015) Rhodiola plants: Chemistry and biological activity. J Food Drug Anal 23(3): 359-369.

18. Myeong SL, Byung Cheul S, Eun JY, Hyun Ja L, Edzard E (2011) Maca (Lepidium meyenii) for treatment of menopausal symtoms: A systematic review. Maturitas 70(3): 227-233.

19. Shruti B, Michael H (2018) Is the hype around the reproductive health claims of maca (Lepidium meyenii Walp.) justified? J Ethnopharmacol 211: $126-170$

20. Gustavo FG (2012) Ethnobiology and ethnopharmacology of Lepidium meyenii (Maca), a plant from the Peruvian highlands. Evid Based Complement Alternat Med 2012: 193496.

21. Sunan W, Fan Z (2019) Chemical composition and health effects of maca (Lepidium meyenii). Food Chem 288: 422-443. 
22. https://8thwondertea.com/products/ashwagandha-apple-chai

24.https://drinkkamsa.com/product/maca-pineapple-coconut/

23. https://www.teslanootropics.com/product-page/nubrain-prodigychild-genius-series

For possible submissions Click below:

Submit Article 